TRANSACTIONS OF THE

AMERICAN MATHEMATICAL SOCIETY

Volume 349, Number 11, November 1997, Pages 4589-4608

S $0002-9947(97) 01917-\mathrm{X}$

\title{
CURVES OF MAXIMUM GENUS IN RANGE A AND STICK-FIGURES
}

\author{
EDOARDO BALLICO, GIORGIO BOLONDI, PHILIPPE ELLIA, \\ AND ROSA MARIA MIRÒ-ROIG
}

\begin{abstract}
In this paper we show the existence of smooth connected space curves not contained in a surface of degree less than $m$, with genus maximal with respect to the degree, in half of the so-called range $\mathrm{A}$. The main tool is a technique of deformation of stick-figures due to G. Fløystad.
\end{abstract}

\section{INTRODUCTION}

A classical problem, which goes back to Halphen $[\mathrm{H}]$, is to determine, for given integers $d$ and $m$, the maximal genus $G(d, m)$ of a smooth projective curve of degree $d$ not contained in a surface of degree $<m$. This problem is actually very natural, and it is in fact one of the cornerstones of the numerical classification of space curves.

The problem of determining $G(d, m)$ depends on the size of $d$ with respect to $m$. Following a long tradition, we distinguish four ranges:

Range $\varnothing$. If $d<\frac{m^{2}+4 m+6}{6}$, then every curve $X$ of degree $d$ satisfies $h^{0}\left(\mathbf{P}^{3}, \mathcal{I}_{X}(m-1)\right) \neq 0$.

Range A. If $\frac{m^{2}+4 m+6}{6} \leq d<\frac{m^{2}+4 m+6}{3}$, then $G(d, m) \leq G_{A}(d, m)$, where $G_{A}(d, m):=1+d(m-1)-\left(\begin{array}{c}m+2 \\ 3\end{array}\right)$.

Range B. 1. If $\frac{m^{2}+4 m+6}{3} \leq d<m^{2}-2 m+2$, then $G(d, m) \geq G_{B}(d, m)$ (see [HH2] for a complete definition of $\left.G_{B}(d, m)\right)$. It is known that $G(d, m)$ is equal to $G_{B}(d, m)$ in several cases (see [Ha2], [GP2], [E], [ES], [S1], [S2]).

2. If $d=m^{2}-2 m+2$, then $G(d, m)=1+(m-3) d$.

Range C. ([GP1] and [GP2]). If $m^{2}-2 m+3 \leq d$, then $G(d, m)$ is exactly known.

In range $\mathrm{A}$, the upper bound for the genus is obtained by using Clifford's theorem (see [Ha1]). A long-standing conjecture is that $G(d, m)=G_{A}(d, m)$; i.e. this genus is actually obtained by some smooth curve.

Two of the present authors announced [BE] an asymptotical solution for it; later on Ch. Walter announced a complete result about curves with seminatural cohomology which implied a positive answer to the conjecture. No written proof has appeared since then, due mainly to the technical nature of both proofs, which heavily used the so-called "méthode d'Horace", which leads to heavy, intricate, and sometimes cumbersome constructions.

Received by the editors March 11, 1996.

1991 Mathematics Subject Classification. Primary 14H50.

Key words and phrases. Stick-figures, maximum genus.

(C)1997 American Mathematical Society 
In this note we give a positive answer to "half" of the problem (see Remark 2.5), with an approach based on the so-called "stick-figures" and which is inspired by the work of Gunnar Fløystad [F] (all techniques are in fact developed in that paper). This construction avoids completely the use of the "méthode d'Horace" by using the smoothing techniques of Hartshorne and Hirschowitz [HH1]. The curves that we find were actually already constructed in [F, 4.3], although their existence is not included in the statement of Fløystad's main theorem 4.10 (see Remark 2.7). Our approach allows us to clearly point out their properties, thus showing immediately that they give a solution to the range A conjecture.

Let us denote

$$
\begin{aligned}
d_{m} & = \begin{cases}\frac{m(m+2)}{4} & \text { if } m \text { is even, } \\
\frac{(m+1)^{2}}{4} & \text { if } m \text { is odd; }\end{cases} \\
D_{m} & =\frac{m(m+1)}{2} .
\end{aligned}
$$

Our main result is (see Corollary 2.4):

Theorem. Let $m$ be an integer, and $d$ such that $d_{m} \leq d \leq D_{m}$. Then there exists a smooth connected curve $X$ of degree $d$ and genus $g=(m-1) d+1-\left(\begin{array}{c}m+2 \\ 3\end{array}\right)$ not contained in any surface of degree less than $m$. In particular, if $d_{m} \leq d<\frac{m^{2}+4 m+6}{3}$, then $G(d, m)=G_{A}(d, m)$.

The construction is simple, once one succeeds in visualizing the combinatorial behaviour of this suitable family of stick-figures with the help of a diagram. In $\S 1$ we discuss some preliminaries: how to draw a diagram carrying the combinatorial properties of the stick-figures that we use; how to check their degree, their genus, their connectedness; the number of nodes lying on every line; and so on.

In $\S 2$ there is the main construction: we choose stick-figures with the needed cohomological properties, and we check that they are smoothable, thus answering the range A problem in the upper half of the interval of degrees.

$\S 3$ is a digression: we discuss the behaviour of the normal bundle of these stickfigures.

In $\S 4$ we show how to modify the construction in order to get curves with genera lower than the maximal possible one ("generalized range A") and such that the restriction map, at the critical value, has maximal rank, and in fact we prove:

Proposition 4.3. Fix an integer $m \geq 2$. For every integer $d, d_{m}<d \leq D_{m}$, there exists a smooth connected curve $X$ such that:

(1) $\operatorname{deg} X=d$,

(2) $H^{1}\left(X, \mathcal{N}_{X}(-1)\right)=0$,

(3) $H^{0}\left(\mathbf{P}^{3}, \mathcal{I}_{X}(m-1)\right)=H^{2}\left(\mathbf{P}^{3}, \mathcal{I}_{Y}(m-1)\right)=0$,

(4) $H^{1}\left(\mathbf{P}^{3}, \mathcal{I}_{X}(m-1)\right)=t$,

(5) $g(X)=G(d, m)-t$,

where $t \in[0, t(d, m)]$ and $t(d, m)=p(2 r-1-p) ; p$ and $r$ are defined in $\S 3$.

A "dual" statement can also be proved:

Proposition 4.4. Fix an integer $m \geq 2$. For every integer $d, d_{m}<d \leq D_{m}$, there exists a smooth connected curve $X$ such that:

(1) $\operatorname{deg} X=d$,

(2) $H^{1}\left(X, \mathcal{N}_{X}(-1)\right)=0$, 
(3) $H^{1}\left(\mathbf{P}^{3}, \mathcal{I}_{X}(m-1)\right)=H^{2}\left(\mathbf{P}^{3}, \mathcal{I}_{Y}(m-1)\right)=0$,

(4) $H^{0}\left(\mathbf{P}^{3}, \mathcal{I}_{X}(m-1)\right)=t$,

(5) $g(X)=G(d, m)+t$

where $t \in\left[0, t^{\prime}(d, m)\right]$ and $t^{\prime}(d, m)=\left(\frac{m}{2}+1-r\right) f ; f$ and $r$ are defined in $\S 4$.

Even if these results are not as complete as those announced by Ballico-Ellia and Walter, we think that it is worthwhile to have them, since this approach is different and the proof is written and, we hope, easy to read.

We thank the referee for his remarks and suggestions, especially for pointing out to us the fact that the stick-figures that we describe in Proposition 2.2 were already constructed by Fløystad in paragraph 4.3 of his paper and for suggesting the questions discussed in the Appendix.

The first, second, and third authors were partially supported by the CNRGNSAGA and the Italian MURST. This project has been strongly encouraged by the European network Europroj, and it has been carried on in the frame of this network. The last author was partially supported by DGICYT PB94-0850.

\section{Generalities}

We will always work in $\mathbf{P}^{3}$, the projective space over an algebraically closed field of characteristic zero.

Definition 1.1. A stick-figure is a reduced union of lines having only simple nodes.

For more details and results about stick-figures the reader is referred to [HH1].

Let $a_{1}=0, a_{2}=0, \ldots, a_{p}=0$, and $b_{1}=0, b_{2}=0, \ldots, b_{p}=0$, be the equations of general planes in $\mathbf{P}^{3}$, and let us denote by $\mathbf{F}_{p}$ the complete intersection stick-figure

$$
a_{1} \cdot a_{2} \cdot a_{p}=0=b_{1} \cdot b_{2} \cdot b_{p} .
$$

We denote by $L_{i j}$ the line $a_{i}=b_{j}=0$. If $Y$ is a sub-stick-figure (where this is taken in the obvious sense) of $\mathbf{F}_{p}$, we denote

$$
\Lambda_{Y}=\left\{(i, j) \mid L_{i j} \subseteq Y\right\} .
$$

Since the planes are chosen generically, then $L_{p q} \cap L_{r s}=\varnothing$ if $p \neq r$ and $q \neq s$. When we need an ordering on the pairs $(i, j)$, we will use the lexicographical order.

Now let $\mathbf{K}_{p}$ be the sub-stick-figure of $\mathbf{F}_{p}$ containing the lines $L_{i j}$ with $i \leq j$, i.e. $\Lambda_{\mathbf{K}_{p}}=\{(i, j) \mid i \leq j\}$. It is easy to prove [F, Lemma 2.11] that $\mathbf{K}_{p}$ has a presentation

$$
0 \rightarrow p \mathcal{O}_{\mathbf{P}^{3}}(-p-1) \stackrel{\Phi}{\rightarrow}(p+1) \mathcal{O}_{\mathbf{P}^{3}}(-p) \rightarrow \mathcal{I}_{\mathbf{K}_{p}} \rightarrow 0
$$

where $\Phi^{t}$ is the matrix of linear forms

$$
\Phi^{t}=\left(\begin{array}{cccccc}
a_{1} & b_{1} & 0 & \cdots & 0 & 0 \\
0 & a_{2} & b_{2} & \cdots & 0 & 0 \\
& & & \cdots & & \\
0 & & & & b_{m-1} & 0 \\
0 & & & & a_{m} & b_{m}
\end{array}\right) .
$$

So, $\mathbf{K}_{p}$ is a degeneration of the well-known family of smooth, arithmetically normal curves having a presentation of the form

$$
0 \rightarrow p \mathcal{O}_{\mathbf{P}^{3}}(-p-1) \rightarrow(p+1) \mathcal{O}_{\mathbf{P}^{3}}(-p) \rightarrow \mathcal{I}_{C} \rightarrow 0 .
$$




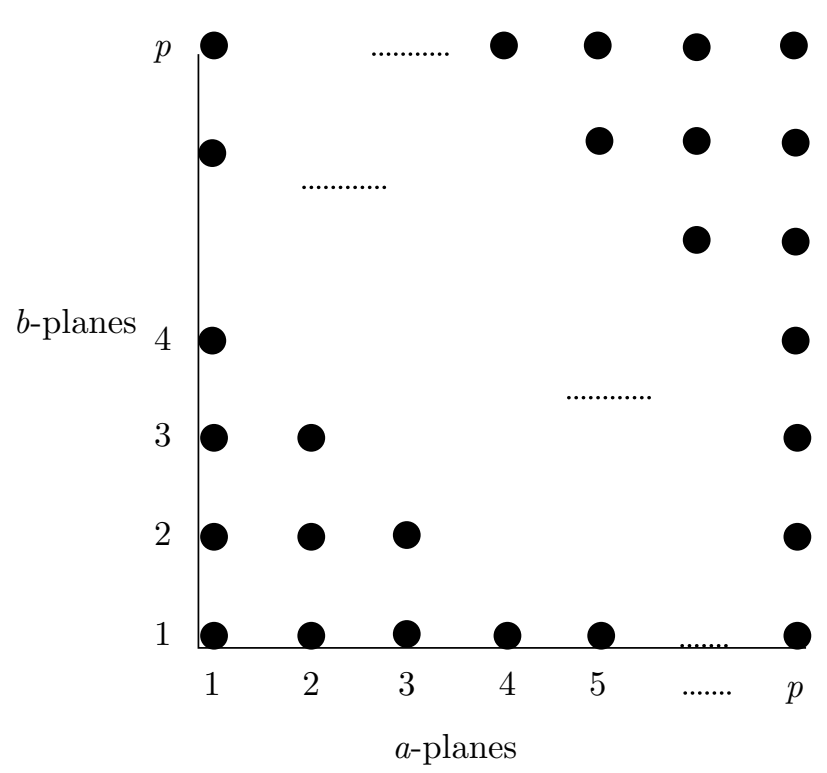

Figure 1

This curve has degree $d=\frac{p(p+1)}{2}$, genus $p_{a}=\frac{p(p+1)(2 p-5)}{6}-1$, and it satisfies

$$
\begin{aligned}
H^{0}\left(\mathbf{P}^{3}, \mathcal{I}_{\mathbf{K}_{p}}(p-1)\right) & =H^{1}\left(\mathbf{P}^{3}, \mathcal{I}_{\mathbf{K}_{p}}(p-1)\right)=H^{2}\left(\mathbf{P}^{3}, \mathcal{I}_{\mathbf{K}_{p}}(p-1)\right)=0, \\
H^{0}\left(\mathbf{P}^{3}, \mathcal{I}_{\mathbf{K}_{p}}(p-2)\right) & =H^{1}\left(\mathbf{P}^{3}, \mathcal{I}_{\mathbf{K}_{p}}(p-2)\right)=H^{2}\left(\mathbf{P}^{3}, \mathcal{I}_{\mathbf{K}_{p}}(p-2)\right)=0, \\
h^{1}\left(\mathbf{Y}, \mathcal{N}_{\mathbf{K}_{p}}(-2)\right) & =0 .
\end{aligned}
$$

In the sequel we will consider only sub-stick-figures of a given $\mathbf{F}_{p}$, chosen as above with $2 p$ general planes. We associate to such a sub-stick-figure $\mathbf{Y}$ a diagram (see Figure 1). In an $\mathbf{N} \times \mathbf{N}$ plane we mark a dot in position $(i, j)$ if $(i, j) \in \Lambda_{Y}$; i.e. if the line $L_{i j}$ is an irreducible component of $\mathbf{Y}$. We call the figure thus obtained the diagram of $\mathbf{Y}$. So, for instance, the diagram of $\mathbf{F}_{p}$ is the $p \times p$ square in Figure 1.

The diagram of $\mathbf{K}_{p}$ is the upper triangle in Figure 2.

The degree of $\mathbf{Y}$ is equal to the number of dots in Figure 2, and two lines meet if and only if the corresponding dots are on the same horizontal or vertical line of the diagram (since the planes $a_{i}$ 's and $b_{j}$ 's are general). This fact gives a simple procedure for counting the number of nodes lying on a line $L_{i j}$ : it is equal to

$N\left(L_{i j}\right):=\#\{\operatorname{dots}$ on the vertical $x=i\}-\#\{\operatorname{dots}$ on the horizontal $y=j\}-2$.

With this remark, the genus of $\mathbf{Y}$ is given by the formula

$$
g(\mathbf{Y})=\frac{\sum_{(i, j) \in \Lambda_{\mathbf{Y}}} N\left(L_{i j}\right)}{2}-d+1
$$

where $d=\operatorname{deg}(\mathbf{T})$.

Remark 1.2. We can easily see from these diagrams an important combinatorial restriction for the stick-figures $\mathbf{Y}$ 's which arise as sub-stick-figures of $\mathbf{F}_{p}$ : if three lines of $\mathbf{Y}$ intersect the same line, then at least two of them must meet. This means, 


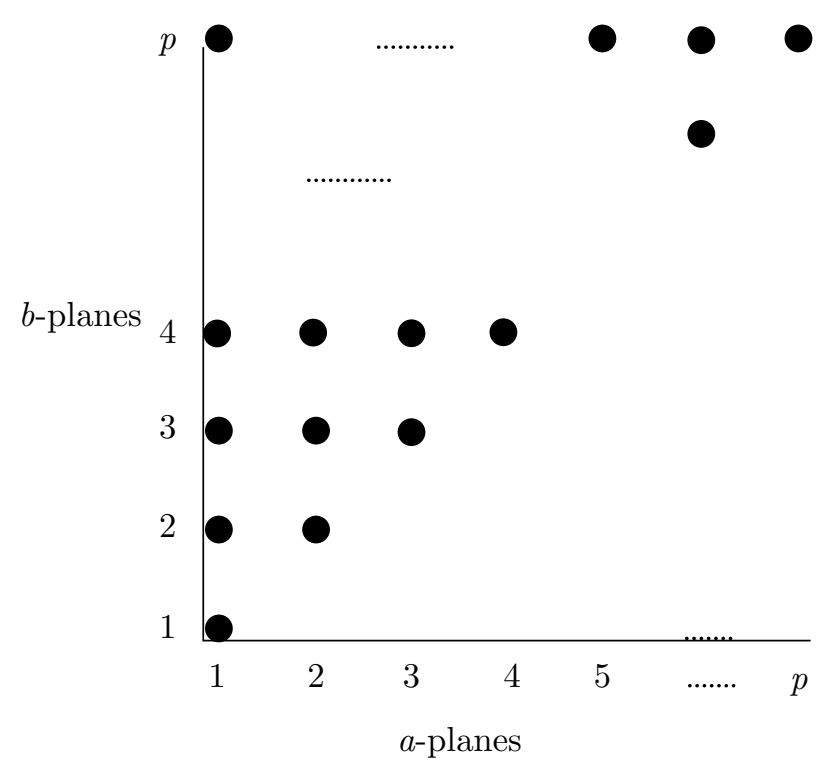

FiguRe 2

for instance, that no "configurations of type $(a, b)$ on a smooth quadric surface", with $b \geq 3$, are allowed.

Remark 1.3. Note that $\mathbf{K}_{p}$ can be assumed to contain a configuration of $p$ lines in general position (the lines $L_{i i}$, with $i=1, \ldots, p$ ).

It is sometimes useful to check if a stick-figure $\mathbf{Y}$ is connected (see for instance [F, Lemma 4.4]). Connectedness may be checked in the diagram of a stick-figure in the following way. Two lines of $\mathbf{Y}$ meet if and only if they are on the same $a$-plane or $b$-plane, i.e. if the corresponding dots are on the same vertical or horizontal line. If $\mathbf{Y}$ is connected, then it is path-connected and it is possible to go from (any point on) any line to (any other point on) any other line. Hence it is possible to find a path along the lines, and "switching of lines" occurs at nodes. That is to say, looking to the diagram of $\mathbf{Y}$, it is possible to go from any dot to any other dot with horizontal or vertical jumps from one dot to another. Conversely, if this property holds, then $\mathbf{Y}$ is path-connected. Hence one may use the

Criterion 1.4. A stick-figure $\mathbf{Y} \subset \mathbf{F}_{p}$ is connected if and only if it is possible to link any dot of the diagram of $\mathbf{Y}$ to any other dot in the diagram via a sequence of horizontal or vertical jumps from one dot to another. (See Figure 3.)

We will need the following easy lemma (which is just a variant for [F, Lemma 4.1 and Lemma 5.1]):

Lemma 1.5. Let $Y \subset \mathbf{P}^{3}$ be a reduced curve, and $L$ and $r$-secant lines intersecting $Y$ quasitransversally. If

$$
H^{0}\left(\mathbf{P}^{3}, \mathcal{I}_{Y \cup L}(r-1)\right)=H^{1}\left(\mathbf{P}^{3}, \mathcal{I}_{Y \cup L}(r-1)\right)=H^{2}\left(\mathbf{P}^{3}, \mathcal{I}_{Y \cup L}(r-1)\right)=0,
$$

then

$$
H^{0}\left(\mathbf{P}^{3}, \mathcal{I}_{Y}(r-1)\right)=H^{1}\left(\mathbf{P}^{3}, \mathcal{I}_{Y}(r-1)\right)=H^{2}\left(\mathbf{P}^{3}, \mathcal{I}_{Y}(r-1)\right)=0,
$$

and vice versa. 
Connected stick-figure. Stick-figure with two connected components.
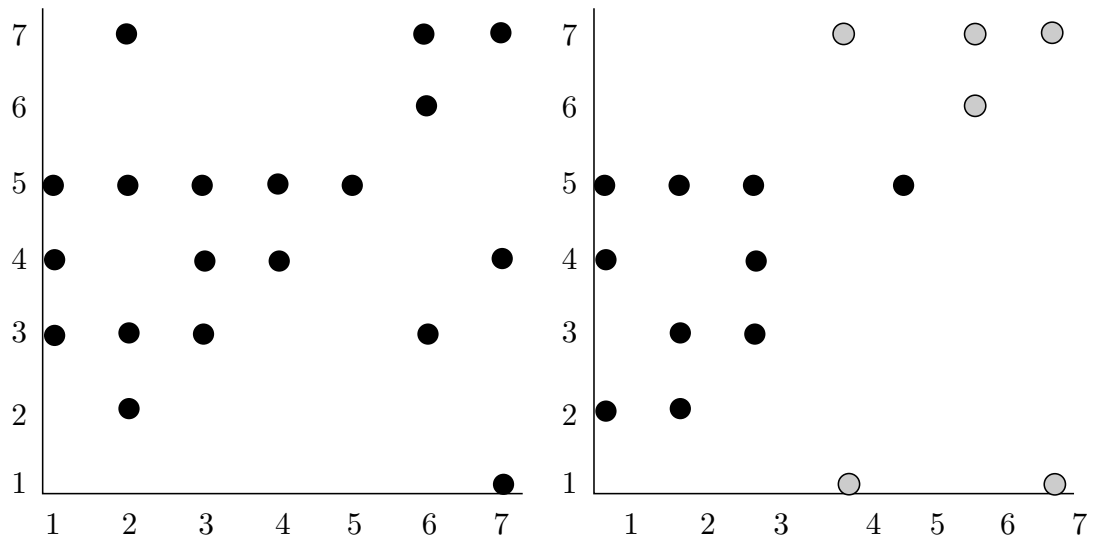

FIGURE 3

Proof. There is an exact sequence

$$
0 \rightarrow \mathcal{I}_{Y \cup L} \rightarrow \mathcal{I}_{Y} \rightarrow \mathcal{I}_{Y / Y \cup L} \rightarrow 0
$$

where $\mathcal{I}_{Y / Y \cup L}=\mathcal{O}_{L}(-r)$. If we tensor (2) with $\mathcal{O}_{\mathbf{P}^{3}}(r-1)$ and we pass to the long cohomology sequence, we get an exact sequence

$$
\begin{aligned}
0 & \rightarrow H^{0}\left(\mathbf{P}^{3}, \mathcal{I}_{Y \cup L}(r-1)\right) \rightarrow H^{0}\left(\mathbf{P}^{3}, \mathcal{I}_{Y}(r-1)\right) \rightarrow H^{0}\left(\mathbf{P}^{3}, \mathcal{O}_{L}(-1)\right) \\
& \rightarrow H^{1}\left(\mathbf{P}^{3}, \mathcal{I}_{Y \cup L}(r-1)\right) \rightarrow H^{1}\left(\mathbf{P}^{3}, \mathcal{I}_{Y}(r-1)\right) \rightarrow H^{1}\left(\mathbf{P}^{3}, \mathcal{O}_{L}(-1)\right) \\
& \rightarrow H^{2}\left(\mathbf{P}^{3}, \mathcal{I}_{Y \cup L}(r-1)\right) \rightarrow H^{2}\left(\mathbf{P}^{3}, \mathcal{I}_{Y}(r-1)\right) \rightarrow 0
\end{aligned}
$$

from which the result follows immediately.

Remark 1.6. This lemma simply means that, given a "good" curve $Y$ of level $r-1$ (in the sense that $h^{i}\left(\mathbf{P}^{3}, \mathcal{I}_{Y}(r-1)\right)=0$ for $\left.i=0,1,2\right)$, it is possible to add or "pick away" $r$-secants (when possible, for instance when $Y$ is a stick-figure), without changing this cohomological vanishing.

Of course, more precise information can be obtained if needed, for instance on the vanishing of cohomology groups for other twists, and this will be done when only some vanishing will be needed.

Now we come to the study of the possibility of smoothing a stick-figure. Since stick-figures are curves with only simple nodes, the main smoothing theorem was proved by Hartshorne and Hirschowitz in [HH1]. It involves the cohomology of (the twists of) the normal bundle of the curve, and the technique for applying it to stick-figures was developed mainly by Fløystad $[\mathrm{F}]$. Hence we describe here his approach, trying to make it easy to handle, via the above diagrams.

Following [F], if $\mathbf{Y} \subset \mathbf{K}_{t}$ is a sub-stick-figure and $L_{p q}$ is a line in $\mathbf{Y}$, we denote

$$
\begin{aligned}
& m_{\mathbf{Y}, a}(p, q)=\#\left\{\left(p, q^{\prime}\right) \in \Lambda_{\mathbf{Y}} \mid p \leq q^{\prime}<q\right\} \\
& m_{\mathbf{Y}, b}(p, q)=\#\left\{\left(p^{\prime}, q\right) \in \Lambda_{\mathbf{Y}} \mid p<p^{\prime} \leq q\right\}
\end{aligned}
$$


and

$$
d_{\mathbf{Y}}(p, q)=m_{\mathbf{Y}, a}(p, q)-m_{\mathbf{Y}, b}(p, q) .
$$

We can visualize $\mathbf{Y}$ as $\mathbf{K}_{t}$ with a certain number of lines taken away. Recall that a node is individuated, in the diagram of $\mathbf{Y}$, by a pair of dots on the same horizontal or vertical line (i.e. two lines on the same $a$-plane or $b$-plane). It will be important to distinguish between the nodes of $\mathbf{F}_{p}$ which are on $\mathbf{Y}$ but which are no longer nodes of $\mathbf{Y}$ from those which are still nodes of $\mathbf{Y}$ : the first ones correspond, in the diagram of $\mathbf{Y}$, to pairs of points with integer coordinates with a "missing" dot, with respect to the "full" square diagram of $\mathbf{F}_{p}$; the other ones correspond to "real" pairs of dots. These integers will count exactly the number of "ghost" pairs and the number of "true" pairs. In fact, how does one compute from the diagram of $\mathbf{Y}$ the numbers $m_{\mathbf{Y}, a}(p, q)$ and $m_{\mathbf{Y}, b}(p, q)$ ? Take the $\operatorname{dot}(p, q)$ and draw the vertical and the horizontal lines starting from $(p, q)$ and meeting the line $i=j$ respectively in the positions $(p, p)$ and $(q, q)$. By definition, $m_{\mathbf{Y}, a}(p, q)$ is simply the number of dots of the "vertical" segment $](p, q),(p, p)]$, and $m_{\mathbf{Y}, b}(p, q)$ is the number of dots of the "horizontal" segment $](p, q),(q, q)]$.

We will perform in $\S 3$ a detailed study of the cohomology of the normal bundle of stick-figures that we construct in $\S 2$ (in particular, we will study the dimension $\left.h^{1}\left(Y, \mathcal{N}_{Y}(-2)\right)\right)$, but in order to smooth them the following lemma is enough:

Lemma 1.7 ([F, Propositions 2.2 and 2.15]). Let $\mathbf{Y} \subset \mathbf{K}_{t}$ be a sub-stick-figure such that

$$
\left|d_{Y}(i, j)\right| \leq 1 \quad \forall(i, j) \in \Lambda_{\mathbf{Y}}
$$

Then $H^{1}\left(\mathbf{Y}, \mathcal{N}_{\mathbf{Y}}(-1)\right)=0$ and therefore $\mathbf{Y}$ is smoothable. If $d_{Y}(i, j)=0 \forall(i, j) \in$ $\Lambda_{\mathbf{Y}}$, then $H^{1}\left(\mathbf{Y}, \mathcal{N}_{\mathbf{Y}}(-2)\right)=0$.

\section{The MAIN CONSTRUCTION}

We will do the construction in full detail for $m$ even; the case $m$ odd is completely analogous. We begin with an easy lemma. Let $\underline{\mathbf{Z}}_{p}=\{1,2, \ldots, p\}$.

Lemma 2.1. Let $A \subseteq \underline{\mathbf{Z}}_{p}, f: A \rightarrow \underline{\mathbf{Z}}_{p}$ be an injective function, and let $\mathbf{X} \subset \mathbf{K}_{p}$ be a stick-figure such that $d_{\mathbf{X}}(i, j)=0 \forall(i, j) \in \Lambda_{\mathbf{X}}$. Then the stick-figure $\mathbf{Y} \subset \mathbf{K}_{p}$ defined by

$$
\Lambda_{\mathbf{Y}}=\Lambda_{\mathbf{X}} \backslash\{(i, f(i)) \mid i \in A\}
$$

satisfies $h^{1}\left(\mathbf{Y}, \mathcal{N}_{\mathbf{Y}}(-1)\right)=0$.

Proof. Let $(p, q) \in \Lambda_{Y}$. Then $m_{\mathbf{Y}, a}(p, q)$ is equal to $m_{\mathbf{X}, a}(p, q)$ if $p \notin A$ or $m_{\mathbf{X}, a}(p, q)-1$ otherwise, and $m_{\mathbf{Y}, b}(p, q)$ is equal to $m_{\mathbf{X}, b}(p, q)$ if $q \notin \operatorname{Im}(f)$ and $m_{\mathbf{Y}, b}(p, q)-1$ otherwise. Hence $|d(p, q)| \leq 1$, and the conclusion follows from Lemma 1.7.

Let us denote

$$
\begin{aligned}
& d_{m}= \begin{cases}\frac{m(m+2)}{4} & \text { if } m \text { is even } \\
\frac{(m+1)^{2}}{4} & \text { if } m \text { is odd; }\end{cases} \\
& D_{m}=\frac{m(m+1)}{2}=\operatorname{deg}\left(\mathbf{K}_{m}\right)
\end{aligned}
$$


Proposition 2.2. Fix an integer $m \geq 2$. For every integer $d, d_{m} \leq d \leq D_{m}$, there exists a smooth connected curve $Z$ of degree $d$, such that

$$
\begin{aligned}
H^{1}\left(Z, \mathcal{N}_{Z}(-1)\right) & =0, \\
H^{0}\left(\mathbf{P}^{3}, \mathcal{I}_{Z}(m-1)\right) & =H^{1}\left(\mathbf{P}^{3}, \mathcal{I}_{Z}(m-1)\right)=H^{2}\left(\mathbf{P}^{3}, \mathcal{I}_{Z}(m-1)\right)=0 .
\end{aligned}
$$

(Note that this is not an asymptotic result.)

Proof. For every $d, d_{m} \leq d \leq D_{m}$, we will exhibit a connected stick-figure $\mathbf{Y}$ (sub-stick-figure of $\mathbf{K}_{m}$ ), satisfying

$$
\begin{aligned}
H^{1}\left(\mathbf{Y}, \mathcal{N}_{\mathbf{Y}}(-1)\right) & =0, \\
H^{0}\left(\mathbf{P}^{3}, \mathcal{I}_{\mathbf{Y}}(m-1)\right) & =H^{1}\left(\mathbf{P}^{3}, \mathcal{I}_{\mathbf{Y}}(m-1)\right)=H^{2}\left(\mathbf{P}^{3}, \mathcal{I}_{\mathbf{Y}}(m-1)\right)=0 .
\end{aligned}
$$

Thanks to Lemma 1.7, $\mathbf{Y}$ will be smoothable, and by semicontinuity the general smooth curve thus obtained will have the same cohomological properties.

Let us fix an integer $m$ even, and let us consider the stick-figure $\mathbf{K}_{m}$. It satisfies

$$
H^{0}\left(\mathbf{P}^{3}, \mathcal{I}_{\mathbf{K} m}(m-1)\right)=H^{1}\left(\mathbf{P}^{3}, \mathcal{I}_{\mathbf{K} m}(m-1)\right)=H^{2}\left(\mathbf{P}^{3}, \mathcal{I}_{\mathbf{K} m}(m-1)\right)=0
$$

and $d(i, j)=0$ for every $(i, j)$.

Let $L$ be a line, irreducible component of $\mathbf{K}_{m}$, where there are $m$ nodes, and let $\mathbf{Y}$ be the stick-figure such that $\mathbf{Y} \cup L=\mathbf{K}_{m}$. Such lines are represented by the dots of the arrangement (in Figure 4) $y=x+1$ (count the dots on the vertical and the horizontal!), and they are mutually disjoint. Hence if we pick away the lines corresponding to the dots $(x, x+1), x=1, \ldots, m-1$, by Lemma 1.5 and Remark 1.6 the residual stick-figures satisfy $H^{i}\left(\mathbf{P}^{3}, \mathcal{I}_{Y}(m-1)\right)=0, i=0,1,2$.

Thanks to Lemma 2.1, whatever amount of these lines we pick away, the residual stick-figure satisfies $H^{1}\left(\mathbf{Y}, \mathcal{N}_{\mathbf{Y}}(-1)\right)=0$, and therefore it is smoothable. The general smooth curve in the family thus obtained satisfies the theorem.

When all the lines corresponding to the dots of the arrangement $y=x+1$ are taken away, we have a stick-figure $\mathbf{X}$, having degree $\frac{m(m+1)}{2}-(m-1)$, such that $d(i, j)=0$ for every $(i, j)$.

But now the dots $(x, x+3), x=1, \ldots, m-3$, represent disjoint lines which are $m$-secant; we can repeat this "picking away". And then we go on as in Figure 4 ; at every step finding a smoothable stick-figure satisfying the two properties $H^{i}\left(\mathbf{P}^{3}, \mathcal{I}_{Y}(m-1)\right)=0, i=0,1,2$, and $h^{1}\left(\mathbf{Y}, \mathcal{N}_{\mathbf{Y}}(-1)\right)=0$.

The degree of $\mathbf{K}_{m}$ is $\frac{m^{2}+m}{2}$, and we can pick away $\left(\frac{m}{2}\right)^{2}$ lines; in this way we find smooth connected curves with the properties

(1) $H^{i}\left(\mathbf{P}^{3}, \mathcal{I}_{Y}(m-1)\right)=0, i=0,1,2$,

(2) $h^{1}\left(\mathbf{Y}, \mathcal{N}_{\mathbf{Y}}(-1)\right)=0$

for every degree $d, \frac{m^{2}+2 m}{4}<d \leq \frac{m^{2}+m}{2}$, and a curve with two connected components for $d=\frac{m^{2}+2 m}{4}$.

In fact, before picking away the last dot, all stick-figures thus obtained are connected. The last one (of degree $\frac{m^{2}+2 m}{4}$ ) has two connected components, as already noted by [F, Lemma 4.4]. One can easily see from Figure 4 that they are in fact two stick-figures of the type $\mathbf{K}_{(m / 2)}$ : white dots correspond to lines that are picked away, black dots to one connected component of the last stick-figure, and grey dots to the other one.

A slightly different strategy of "picking away lines" is needed if we want also a smooth connected curve of degree $\frac{m^{2}+m}{4}$, satisfying $H^{i}\left(\mathbf{P}^{3}, \mathcal{I}_{\mathbf{Y}}(m-1)\right)=0$, 


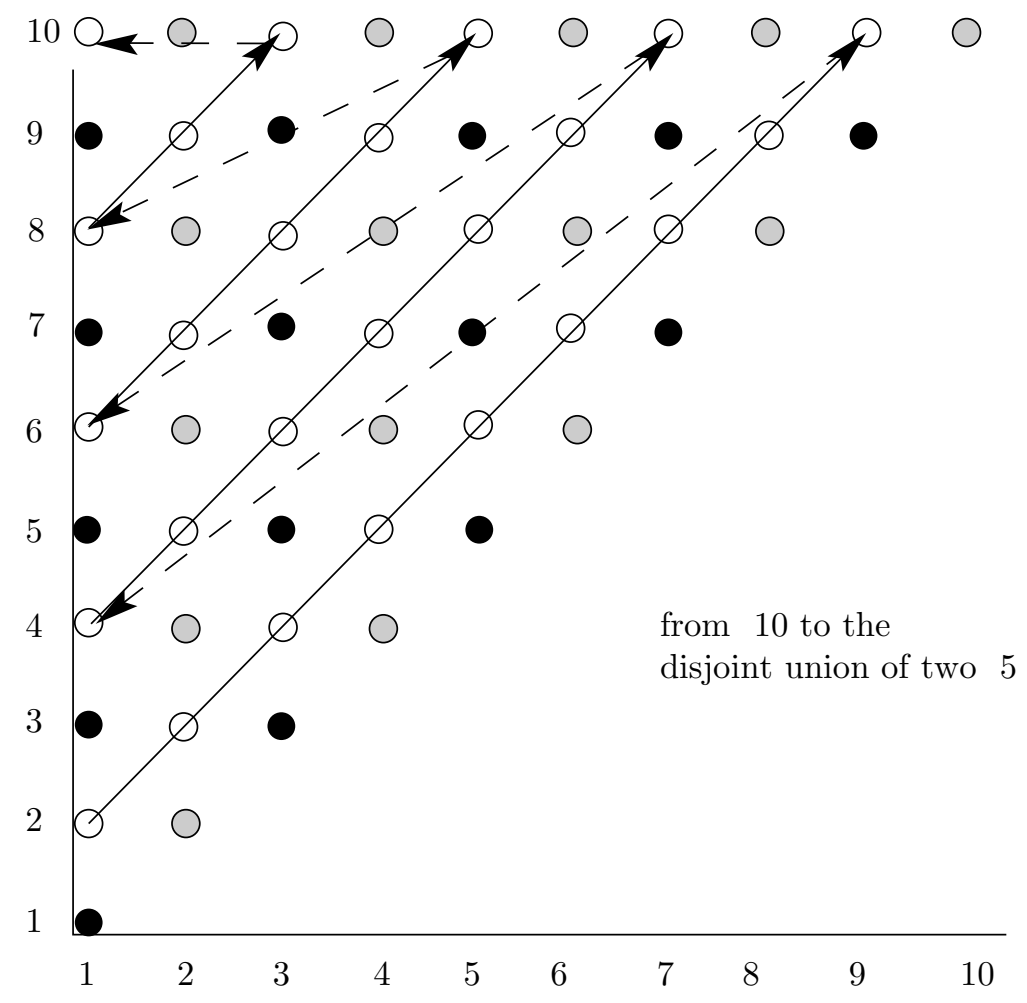

FigURE 4

$i=0,1,2$. Simply, follow the same "path" for picking away lines, but take away the $\operatorname{dot}(m-2, m)$ instead of the $\operatorname{dot}(m-1, m)$. An easy check (by using Criterion 1.4) proves the connectedness of the stick-figure. (See Figure 5.)

Remark 2.3. Let us denote by $\mathbf{X}_{m[(m / 2)-1]}$ the stick-figure obtained after removing from $\mathbf{K}_{m}$ all the dots $(x, y)$ satisfying $y=x+1$, by $\mathbf{X}_{m[(m / 2)-2]}$ the stick-figure obtained after removing from $\mathbf{X}_{m[(m / 2)-1]}$ all the dots $(x, y)$ satisfying $y=x+3$, and in general by $\mathbf{X}_{m(r-1)}$ the stick-figure obtained after removing from $\mathbf{X}_{m r}$ all the dots $(x, y)$ satisfying $y=x+m-2 r+1,1 \leq r \leq(m / 2)-1$.

These stick-figures have $h^{1}\left(\mathbf{Y}, \mathcal{N}_{\mathbf{Y}}(-2)\right)=0$, since they satisfy $d(i, j)=0$ for every $(i, j) \in \Lambda_{\mathbf{Y}}$. An easy computation shows that

$$
\operatorname{deg}\left(X_{m r}\right)=\frac{m^{2}+m}{4}+r^{2}
$$

This is exactly the degree of the disjoint union $\mathbf{K}_{(m / 2)-r} \bullet \mathbf{K}_{(m / 2)+r}$.

Corollary 2.4 (upper half of the range A). Let $m$ be an integer, and $d$ such that $d_{m} \leq d \leq D_{m}$. Then there exists a smooth connected curve $X$ of degree $d$ and genus $g=(m-1) d+1-\left(\begin{array}{c}m+2 \\ 3\end{array}\right)$ not contained in any surface of degree less than $m$.

Proof. Let $X$ be the smooth curve constructed in Proposition 2.2. We have $\chi\left(\mathcal{I}_{\mathbf{X}}(m-1)\right)=0$; hence the result follows from the Riemann-Roch theorem.

Remark 2.5. From the corollary above, we can see that more than half of the curves of maximal genus in the range A can be obtained with this procedure by smoothing 


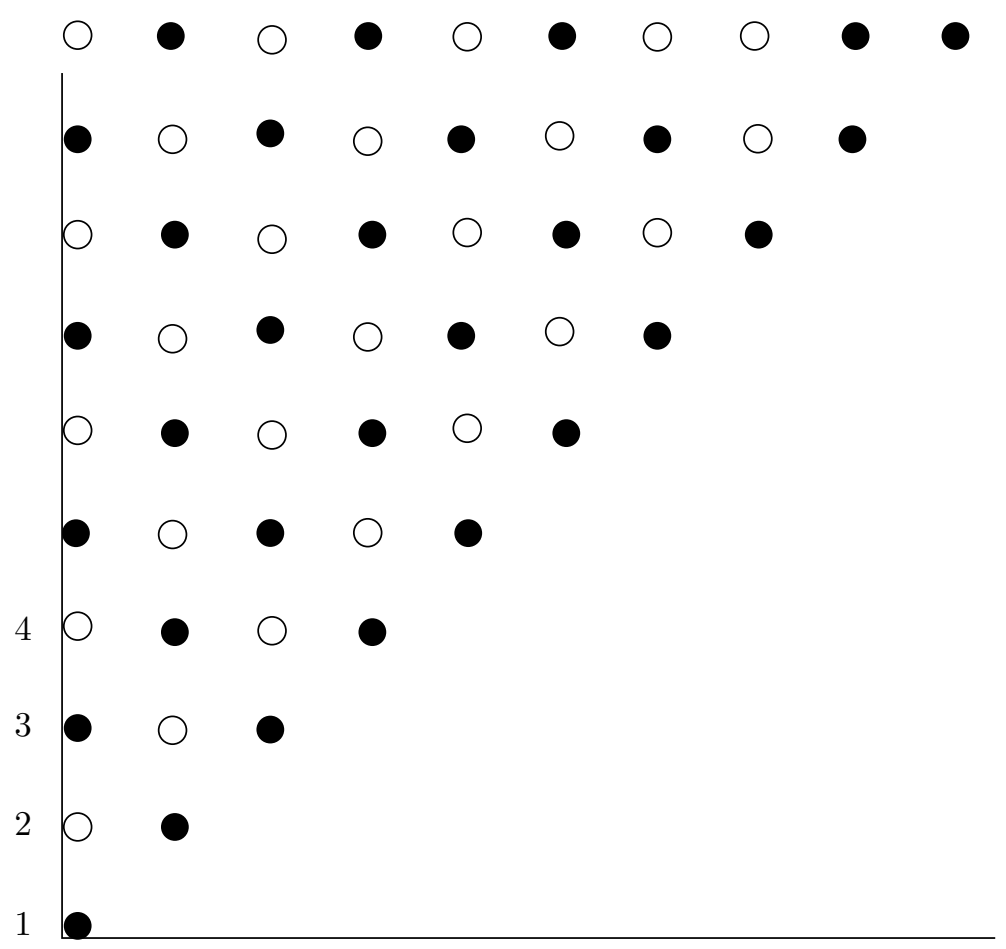

Figure 5

suitable stick-figures: the middle of the interval

$$
\left[\frac{m^{2}+4 m+6}{6}, \frac{m^{2}+4 m+6}{3}[\right.
$$

occurs in fact for $\frac{m^{2}+4 m+6}{4}$.

Remark 2.6. Clearly these curves have maximal rank: it follows from an easy argument as in Lemma 1.5.

Remark 2.7. In paragraph 4.3 of his paper [F], Fløystad gives an algorithm for picking away from $\mathbf{K}_{m} s$ lines which at the step of their removal are $(m-1)$ subsecant, and $t$ lines which are $m$-subsecant, whenever $s+t \leq \frac{m^{2}-p_{m}}{4}$, where $p_{m}$ is 1 if $m$ is odd, 0 otherwise. So, if we just consider the case $s=0$, this is exactly the procedure that we explained above (we will show in the Appendix how to see Fløystad's paragraph 4.3 in our diagrams, explaining the way for pushing it a little further).

But while expressing the numerical results of this procedure, many of these curves are somehow "forgotten" in Fløystad's paper. We will first give a numerical example, and then we will explain the reason for this.

Theorem 4.10 of $[\mathrm{F}]$ states that for all pairs of integers $(d, g)$ such that $d \geq 1$ and $C_{K}(d) \geq g \geq C_{1}(d)$, there are smooth connected curves $Y$ with degree $d$ and genus $g$ having maximal rank (and other properties). 


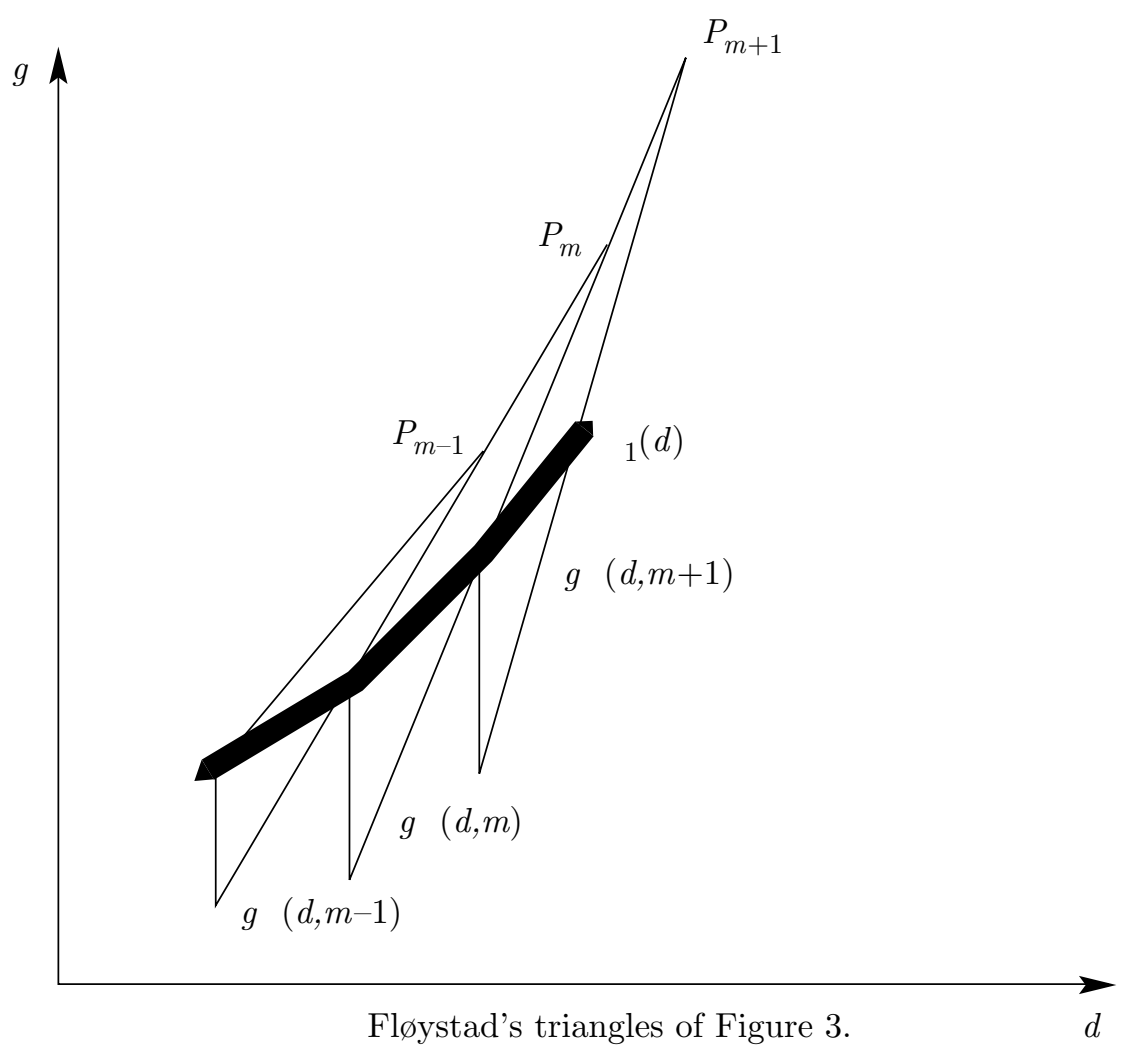

FiguRE 6

Take for instance $d=20$. Then $C_{K}(20)=46$ and $C_{1}(20)=37$. So Fløystad's main theorem 4.10 states the existence of smooth connected curves of degree 20 and genus between 37 and 46 .

But now look to the range A problem for $m=8$. In this case, $\frac{m(m+2)}{4}=20$, and $\frac{m^{2}+4 m+6}{3}=34$; hence the construction of Proposition 2.2 gives a curve of degree 20 and genus $21<C_{1}(20)$. In the same way, the curves of degrees $21,22,23, \ldots$ and genera $G_{A}(21,8), G_{A}(22,8), G_{A}(23,8), \ldots$, respectively, are not included in Theorem 4.10 of $[\mathrm{F}]$. (See Figure 6.)

The reason for this is simple. Figure 3 in Fløystad's paper gives the set of pairs $(d, g)$ of stick-figures obtained by $\mathbf{K}_{m}$ by removing $(m-1)$-secants and $m$-secants, as explained before. The curves that we use for solving the problem of maximum genus in range $\mathrm{A}$ correspond to the $P_{m} P_{m}^{2}$ side of the triangle.

The contribution from different $\mathbf{K}_{m}$ 's are patched together in Fløystad's Figure 4 , where we marked with a bold line the curve $C_{1}(d)$. So we clearly see why many of the curves that we are interested in are forgotten in the statement of Theorem 4.10 of $[\mathrm{F}]$.

For a more detailed discussion of Fløystad's Figures 3 and 4 in terms of our diagrams see the Appendix. 


\section{Cohomological behaviour of $\mathcal{N}_{\mathbf{Y}}(-2)$}

In this section, which is independent from the other parts of the paper, we will perform a detailed study of $H^{1}\left(\mathbf{Y}, \mathcal{N}_{\mathbf{Y}}(-2)\right)$, where $Y$ is a stick-figure arising from the construction of Proposition 2.2. It is possible to see exactly where the obstructions to the vanishing of this group are. We need to recall here the details of Fløystad's approach to the description of the normal bundle of a stick-figure (in fact, the first part of this paragraph is nothing but a re-writing of part of [F, $\S 2]$ that we need in order to make understandable what follows), and we will reduce the computation of the dimension $h^{1}\left(\mathbf{Y}, \mathcal{N}_{\mathbf{Y}}(-2)\right)$ to a combinatorial study of the stick-figure $\mathbf{Y}$. We follow (up to some minor changes) wherever possible Fløystad's notation. The main idea is to decompose the cohomology groups involved in order to point out the "contribution" of each node and each line to $h^{1}\left(\mathbf{Y}, \mathcal{N}_{\mathbf{Y}}(-2)\right)$.

As in $\S 1$, let $a_{1}=0, a_{2}=0, \ldots, a_{p}=0$ (" $a$-planes") and $b_{1}=0, b_{2}=0, \ldots, b_{p}=0$ ("b-planes") be the equations of general planes in $\mathbf{P}^{3}$, and let us denote by $\mathbf{F}_{p}$ the complete intersection stick-figure given by the equations $a_{1} \cdot a_{2} \cdot a_{p}=0=b_{1} \cdot b_{2} \cdot b_{p}$. Let $\mathbf{Y} \subset \mathbf{F}_{p}$ be a sub-stick-figure. Its nodes are divided into two sets:

$a$-nodes: $a$-nodes are those nodes which are the intersection of two lines lying on an $a$-plane. We denote by $P_{i ;(j, k)}$ the node given by the intersection of the lines $L_{i j}$ and $L_{i k}$; these lines lie on the $a$-plane $a_{i}=0$. We denote by $S_{\mathbf{Y}, a}$ the set of $a$-nodes of $\mathbf{Y}$.

$b$-nodes: $b$-nodes are those nodes which are the intersection of two lines on a $b$-plane; we denote by $P_{(i, 1) ; j}$ the node $L_{i j} \cap L_{l j}$, and by $S_{\mathbf{Y}, b}$ the set of $b$-nodes.

We will use a suitable natural splitting of $\mathcal{N}_{\mathbf{Y}}$. Recall that the stick-figure $\mathbf{Y}$ is a sub-stick-figure of $\mathbf{F}_{p}$, where $\mathbf{F}_{p}=A \cap B$, and $A$ is the reducible surface of degree $p$ whose equation is $a_{1} \cdot a_{2} \cdots a_{p}=0$, and $B$ is the reducible surface of degree $p$ whose equation is $b_{1} \cdot b_{2} \cdots b_{p}=0$. Hence the normal bundle of $\mathbf{F}_{p}$ splits:

$$
\mathcal{N}_{\mathbf{F}_{p}} \cong \mathcal{N}_{\mathbf{F}_{p} / A} \oplus \mathcal{N}_{\mathbf{F}_{p} / B},
$$

where $\mathcal{N}_{\mathbf{F}_{p} / A}$ (resp. $\left.\mathcal{N}_{\mathbf{F}_{p} / B}\right)$ is the normal bundle of $\mathbf{F}_{p}$ in $A$ (resp. in $B$ ), which we will denote by $L_{A}$ (resp. by $L_{B}$ ).

Now if $X$ is a curve with isolated singularities, let $T_{X}^{1}=\bigoplus_{P \in \operatorname{Sing} X} T_{X, P}^{1}$ be the cokernel of the natural map $\mathcal{T}_{\mathbf{P}^{3}} \otimes \mathcal{O}_{X} \rightarrow \mathcal{N}_{\mathbf{X}}$. This sheaf has support on the singularities of $X$, and $T_{X, P}^{1}$ has length one if $P$ is a single node, as in our situation (for more details about $T_{X}^{1}$ the reader is referred to [HH1]). If $G$ is a subset of Sing $X$, we will write $T_{X, G}^{1}=\bigoplus_{P \in G} T_{X, P}^{1}$.

Hence, if we divide the nodes of $\mathbf{F}_{p}$ into the set $S_{a}$ of $a$-nodes and the set $S_{b}$ of $b$-nodes, the sheaf $T_{\mathbf{F}_{p}}^{1}$ splits as $T_{\mathbf{F}_{p}}^{1} \cong T_{\mathbf{F}_{p}, S_{a}}^{1} \oplus T_{\mathbf{F}_{p}, S_{b}}^{1}$.

In the natural map $\mathcal{N}_{\mathbf{F}_{p}} \rightarrow T_{\mathbf{F}_{p}}^{1}$, which can be written as

$$
L_{A} \oplus L_{B} \rightarrow T_{\mathbf{F}_{p}, S_{a}}^{1} \oplus T_{\mathbf{F}_{p}, S_{b}}^{1},
$$

a direct check gives that the maps

$$
L_{A} \rightarrow T_{\mathbf{F}_{p}, S_{b}}^{1} \text { and } L_{B} \rightarrow T_{\mathbf{F}_{p}, S_{a}}^{1}
$$

are zero.

If $\mathbf{Y}$ is a sub-stick-figure of $\mathbf{F}_{p}$, from [F, Lemma 1.3], we get an exact sequence

$$
0 \rightarrow \mathcal{N}_{\mathbf{Y}} \rightarrow \mathcal{N}_{\mathbf{F}_{p} \mid \mathbf{Y}} \rightarrow T_{\mathbf{F}_{p}, R}^{1} \rightarrow 0
$$


where $R$ is the set $R:=\left[\operatorname{Sing}\left(\mathbf{F}_{p}\right) \cap \mathbf{Y}\right] \backslash \operatorname{Sing}(\mathbf{Y})$. As usual, we can divide $R$ into $a$-nodes and $b$-nodes: $R=R_{a} \cup R_{b}$, and the sequence becomes

$$
0 \rightarrow \mathcal{N}_{Y} \rightarrow L_{A \mid \mathbf{Y}} \oplus L_{B \mid \mathbf{Y}} \rightarrow T_{\mathbf{F}_{p}, R_{a}}^{1} \oplus T_{\mathbf{F}_{p}, R_{b}}^{1} \rightarrow 0,
$$

and $\mathcal{N}_{\mathbf{Y}}$ splits as $\mathcal{N}_{\mathbf{Y}} \cong K_{A} \oplus K_{B}$, where

$$
K_{A}=\operatorname{ker}\left(L_{A \mid Y} \rightarrow T_{\mathbf{F}_{p}, R_{a}}^{1}\right), K_{B}=\operatorname{ker}\left(L_{B \mid \mathbf{Y}} \rightarrow T_{\mathbf{F}_{p}, R_{b}}^{1}\right) .
$$

Hence the sequence $(*)$ splits as

$$
0 \rightarrow K_{A} \oplus K_{B} \rightarrow L_{A \mid \mathbf{Y}} \oplus L_{B \mid \mathbf{Y}} \rightarrow T_{\mathbf{F}_{p}, R_{a}}^{1} \oplus T_{\mathbf{F}_{p}, R_{b}}^{1} \rightarrow 0 .
$$

Hence the long exact sequences to be studied are

$$
\begin{aligned}
0 & \rightarrow H^{0}\left(\mathbf{Y}, K_{B}(-2)\right) \rightarrow H^{0}\left(\mathbf{Y}, L_{B \mid \mathbf{Y}}(-2)\right) \rightarrow H^{0}\left(\mathbf{Y}, T_{\mathbf{F}_{p}, R_{b}}^{1}\right) \\
& \rightarrow H^{1}\left(\mathbf{Y}, K_{B}(-2)\right) \rightarrow H^{1}\left(\mathbf{Y}, L_{B \mid \mathbf{Y}}(-2)\right) \rightarrow 0
\end{aligned}
$$

and

$$
\begin{aligned}
0 & \rightarrow H^{0}\left(\mathbf{Y}, K_{A}(-2)\right) \rightarrow H^{0}\left(\mathbf{Y}, L_{A \mid \mathbf{Y}}(-2)\right) \rightarrow H^{0}\left(\mathbf{Y}, T_{\mathbf{F}_{p}, R_{a}}^{1}\right) \\
& \rightarrow H^{1}\left(\mathbf{Y}, K_{A}(-2)\right) \rightarrow H^{1}\left(\mathbf{Y}, L_{A \mid \mathbf{Y}}(-2)\right) \rightarrow 0,
\end{aligned}
$$

and in particular we want to give a bound for

$$
\begin{aligned}
& h^{1}\left(\mathbf{Y}, K_{B}(-2)\right) \\
& \quad=h^{1}\left(\mathbf{Y}, L_{B \mid \mathbf{Y}}(-2)\right)+\operatorname{dimcoker}\left[H^{0}\left(\mathbf{Y}, L_{B \mid \mathbf{Y}}(-2)\right) \rightarrow H^{0}\left(\mathbf{Y}, T_{\mathbf{F}_{p}, R_{b}}^{1}\right)\right]
\end{aligned}
$$

and

$$
\begin{aligned}
& h^{1}\left(\mathbf{Y}, K_{A}(-2)\right) \\
& =h^{1}\left(\mathbf{Y}, L_{A \mid \mathbf{Y}}(-2)\right)+\operatorname{dimcoker}\left[H^{0}\left(\mathbf{Y}, L_{A \mid \mathbf{Y}}(-2)\right) \rightarrow H^{0}\left(\mathbf{Y}, T_{\mathbf{F}_{p}, R_{a}}^{1}\right)\right] .
\end{aligned}
$$

Let us study the first group. As in $[\mathrm{F}, \S 2.7]$, one finds a commutative diagram

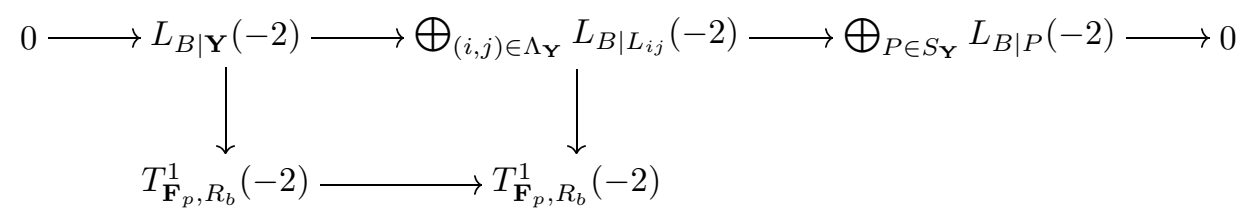

from which we get (in $[\mathrm{F}]$ there is an explicit description of the maps) that

$$
\begin{aligned}
h^{1}\left(\mathbf{Y}, K_{B}(-2)\right)=\operatorname{dimcoker}[ & \bigoplus_{(i, j) \in \Lambda_{\mathbf{Y}}} H^{0}\left(\mathbf{Y}, L_{B \mid L_{i j}}(-2)\right) \\
& \left.\rightarrow \bigoplus_{P \in S_{\mathbf{Y}}} H^{0}\left(\mathbf{Y}, L_{B \mid P}(-2)\right) \oplus H^{0}\left(\mathbf{Y}, T_{\mathbf{F}_{p}, R_{b}}^{1}(-2)\right)\right] .
\end{aligned}
$$

For fixed $(i, j) \in \Lambda_{\mathbf{Y}}$, the vector space $H^{0}\left(\mathbf{Y}^{\prime}, L_{B \mid L_{i j}}(-2)\right)$ is generated by the images of the forms

$$
a_{i}^{s}=a_{1} \cdot a_{2} \cdots \hat{a}_{s} \cdots \hat{a}_{i} \cdots a_{p} \in H^{0}\left(\mathbf{P}^{3}, \mathcal{O}_{\mathbf{P}^{3}}(p-2)\right),
$$


which are in fact a basis; we denote these images by $a_{i j}^{s}$. Hence we decompose

$$
F=\bigoplus_{(i, j) \in \Lambda_{\mathbf{Y}}} H^{0}\left(\mathbf{Y}, L_{B \mid L_{i j}}(-2)\right)
$$

as $F_{1} \oplus F_{2}$, where $F_{1}$ is generated by the forms $a_{i j}^{s}$ for which the $b$-node $P_{(i, s) ; j}=$ $L_{i j} \cap L_{s j}$ belongs to $R_{\mathbf{Y}, b}$, and $F_{2}$ is generated by the forms $a_{i j}^{s}$ for which the node $P_{(i, s) ; j}$ belongs to $S_{\mathbf{Y}}$.

In other words, $F_{1}$ is generated by the forms $a_{i j}^{s}$ for which the line $L_{s j} \notin \mathbf{Y}$, and $F_{2}$ is generated by the forms $a_{i j}^{s}$ for which the line $L_{s j} \in \mathbf{Y}$.

We also decompose the image $\bigoplus_{P \in S_{\mathbf{Y}}} H^{0}\left(\mathbf{Y}, L_{B \mid P}(-2)\right) \oplus H^{0}\left(\mathbf{Y}, T_{\mathbf{F}_{b}, R_{b}}^{1}(-2)\right)$ as

$$
\left[\bigoplus_{P \in S_{\mathbf{Y}, a}} H^{0}\left(\mathbf{Y}, L_{B \mid P}(-2)\right)\right] \oplus\left[\bigoplus_{P \in S_{\mathbf{Y}, b}} H^{0}\left(\mathbf{Y}, L_{B \mid P}(-2)\right)\right] \oplus H^{0}\left(\mathbf{Y}, T_{\mathbf{F}_{p}, R_{b}}^{1}(-2)\right),
$$

that we simply will write $H_{a} \oplus H_{b} \oplus T$.

A direct check shows that $F_{1} \rightarrow T$ is an isomorphism and $F_{2} \rightarrow T$ is the zero map; hence $\operatorname{dim}\left(\operatorname{coker}\left[F_{1} \oplus F_{2} \rightarrow H_{a} \oplus H_{b} \oplus T\right]\right)=\operatorname{dim}\left(\operatorname{coker}\left[F_{2} \rightarrow H_{a} \oplus H_{b}\right]\right)$.

Again as in [F, §2.9], we decompose $F_{2}$ as $G_{1} \oplus G_{2}$, where $G_{1}$ is the subspace generated by the forms $v_{i i^{\prime}, j}=a_{i j}^{i^{\prime}}+a_{i^{\prime} j}^{i}$, where $i, i^{\prime}$ and $j$ are such that the node $P_{\left(i, i^{\prime}\right) ; j} \in S_{\mathbf{Y}}$, and $G_{2}$ is the subspace generated by the forms $a_{i j}^{i^{\prime}}$ for which the node $P_{\left(i, i^{\prime}\right) ; j} \in S_{\mathbf{Y}}$, and $i<i^{\prime}$.

As above, the map $G_{2} \rightarrow H_{b}$ is an isomorphism and $G_{1} \rightarrow H_{b}$ is zero; hence

$$
\operatorname{dim}\left(\operatorname{coker}\left[F_{2} \rightarrow H_{a} \oplus H_{b}\right]\right)=\operatorname{dim}\left(\operatorname{coker}\left[G_{2} \rightarrow H_{a}\right]\right) .
$$

If we denote by $V_{i j}$ the space generated by the forms $v_{i i^{\prime}, j} \in G_{1}$ such that $i<$ $i^{\prime} \leq j$, then $\operatorname{dim} V_{i j}=m_{\mathbf{Y}, b}(i, j)$ and $G_{1}=\bigoplus_{(i, j) \in \Lambda_{\mathbf{Y}}} V_{i j}$. But we have a similar decomposition of $H_{a}$ as a direct sum $\bigoplus_{(i, j) \in \Lambda_{\mathbf{Y}}} H_{a i j}$, where

$$
H_{a i j}=\bigoplus_{i \leq j^{\prime}<j, P_{i ;\left(j^{\prime} j\right)} \in S_{\mathbf{Y} a}} H^{0}\left(\mathbf{Y}, L_{B \mid P_{i ;\left(j^{\prime} j\right)}}(-2)\right) .
$$

One may check that $\operatorname{dim} H_{a i j}=m_{\mathbf{Y}, a}(i, j)$. If we give to $\Lambda_{\mathbf{Y}}$ the lexicographical order, then $[\mathrm{F}, \S 2.12] \Phi\left(V_{i j}\right)$ is contained in $\bigoplus_{\left(i^{\prime}, j^{\prime}\right) \geq(i, j)} H_{a i^{\prime} j^{\prime}}$; hence we get flags of vector spaces which are respected by $\Phi$. But now from [F, Lemma 1.6] we have that the maps $\Phi_{i j}: V_{i j} \rightarrow H_{a i j}$ have maximal ranks; hence

$$
\operatorname{dim}\left(\operatorname{coker}\left[V_{i j} \rightarrow H_{a i j}\right]\right)=\max \left\{m_{\mathbf{Y}, a}(i, j)-m_{\mathbf{Y}, b}(i, j), 0\right\}=\max \{d(i, j), 0\} .
$$

From a flag-type lemma we get again that $\operatorname{dim}(\operatorname{coker} \Phi) \leq \sum_{(i, j) \in \Lambda_{\mathbf{Y}}} \operatorname{dim}\left(\operatorname{coker} \Phi_{i j}\right)$.

From our construction, it follows (for our stick-figures constructed in $\S 2$ ) that $|d(i, j)| \leq 1$; hence every line $L_{i j}$ for which $d(i, j) \neq 0$ gives a contribution of dimension 1 to $\sum_{(i, j) \in \Lambda_{\mathbf{Y}}} \operatorname{dim}\left(\operatorname{coker} \Phi_{i j}\right)$ (either to the $A$-part or to the $B$-part, depending on the sign of $d(i, j))$. Hence $\sum_{(i, j) \in \Lambda_{\mathbf{Y}}} \operatorname{dim}\left(\operatorname{coker} \Phi_{i j}\right)$ is equal to the number of lines for which $d(i, j) \neq 0$.

But now, let us consider the stick-figure $\mathbf{Y}$ of degree $d=d_{m(r-1)}+p$ constructed in $\S 2$. Its diagram is obtained from the diagram of $\mathbf{K}_{m}$ by picking away all the dots of the arrangements $y=x+1, y=x+3, \ldots, y=x+m-2 r-1$, and $(2 r-1-p)$ dots of the arrangement $y=x+m-2 r+1$. Hence the number of lines for which $d(i, j) \neq 0$ is equal to the number of dots in the upper-left rectangle 


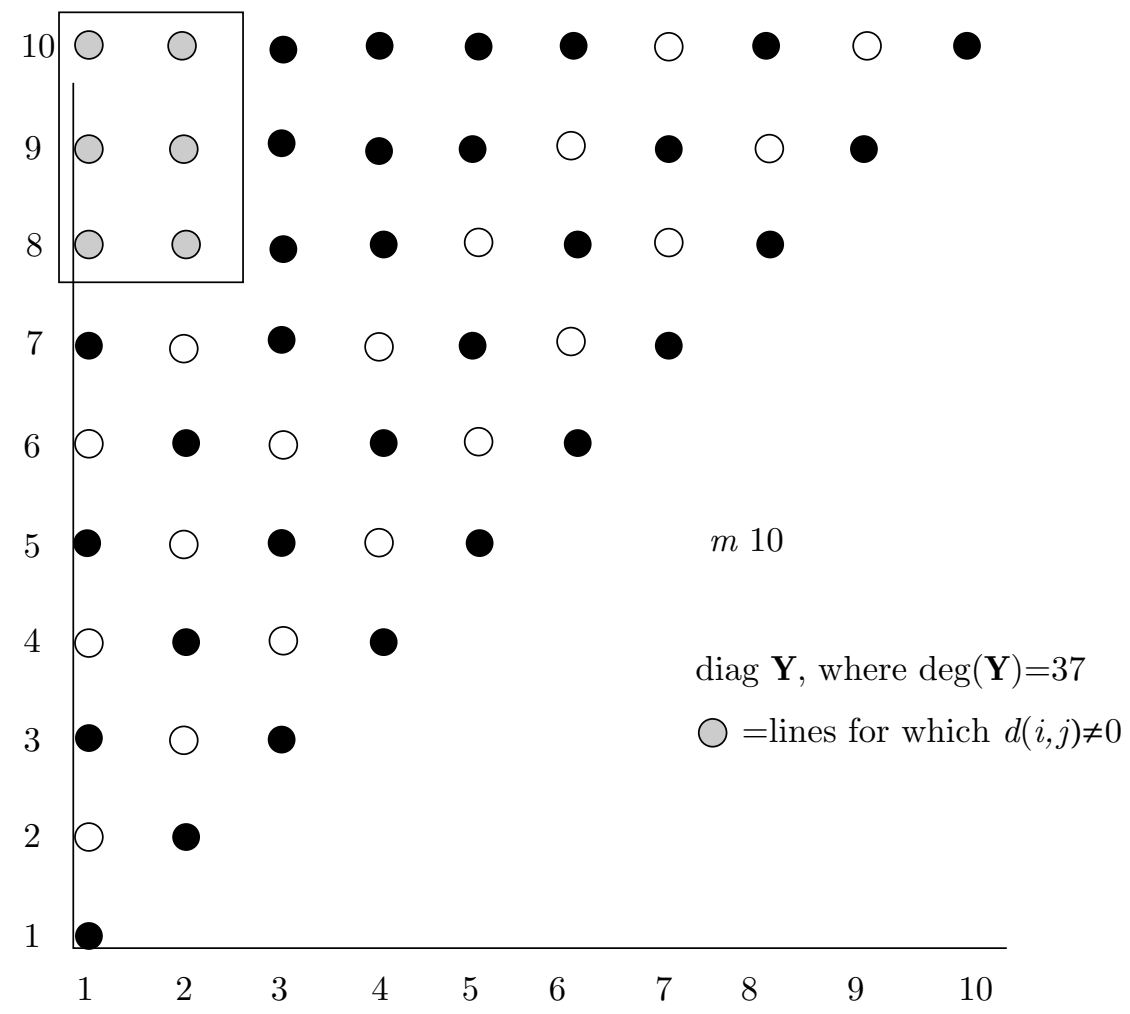

FigURE 7

(see Figure 7$)$, i.e. to $p(2 r-1-p)$. Hence $h^{1}\left(\mathbf{Y}, \mathcal{N}_{\mathbf{Y}}(-2)\right) \leq p(2 r-1-p)$, where $p=\operatorname{deg}(\mathbf{Y})-d_{m(r-1)}$.

\section{Generalized range A (upper half part)}

Our main proposition (Corollary 2.4) gives the existence of smooth, connected curves of the maximal possible genus, for a given degree in a certain range, not lying on a surface of degree $m-1$. This was obtained by first constructing a suitable stick-figure $\mathbf{Y}$, that was proved to be smoothable, with

$$
H^{0}\left(\mathbf{P}^{3}, \mathcal{I}_{\mathbf{Y}}(m-1)\right)=H^{1}\left(\mathbf{P}^{3}, \mathcal{I}_{\mathbf{Y}}(m-1)\right)=H^{2}\left(\mathbf{P}^{3}, \mathcal{I}_{\mathbf{Y}}(m-1)\right)=0 .
$$

Let us denote the degree of $\mathbf{Y}$ by $d$ and the arithmetic genus of $\mathbf{Y}$ by $G(d, m)$ (as customary); note that $G(d, m)=1+d(m-1)-\left(\begin{array}{c}m+2 \\ 3\end{array}\right)$. In particular, for this stick-figure, the restriction map

$$
\rho_{\mathbf{Y}}(m-1): H^{0}\left(\mathbf{P}^{3}, \mathcal{O}_{\mathbf{P}^{3}}(m-1)\right) \rightarrow H^{1}\left(\mathbf{P}^{3}, \mathcal{O}_{\mathbf{Y}}(m-1)\right)
$$

is bijective.

With some changes in the proof, the same construction will give the existence of smoothable stick-figures, for fixed $m$ and $d$, such that

$$
H^{0}\left(\mathbf{P}^{3}, \mathcal{I}_{\mathbf{Y}}(m-1)\right)=H^{2}\left(\mathbf{P}^{3}, \mathcal{I}_{\mathbf{Y}}(m-1)\right)=0
$$

and $h^{1}\left(\mathbf{P}^{3}, \mathcal{I}_{\mathbf{Y}}(m-1)\right)=t$, for every $t$ in a certain range $[0, t(d, m)]$. 
This means, in other terms, that $\rho_{\mathbf{Y}}(m-1)$ is injective and the genus of $\mathbf{Y}$ is exactly $G(d, m)-t$. So we find some genera below the maximal possible genus, for curves not lying on a surface of degree less than $m$. This construction is related also to $[\mathrm{F}, 5.6]$.

We first need another version of Lemma 1.5.

Lemma 4.1. Let $Y \subset \mathbf{P}^{3}$ be a reduced curve, and $L$ and $g$-secant lines intersecting $X$ quasitransversally. If

$$
H^{0}\left(\mathbf{P}^{3}, \mathcal{I}_{Y \cup L}(r-1)\right)=H^{2}\left(\mathbf{P}^{3}, \mathcal{I}_{Y \cup L}(r-1)\right)=0
$$

and $g \geq r$, then

$$
H^{0}\left(\mathbf{P}^{3}, \mathcal{I}_{Y}(r-1)\right)=H^{2}\left(\mathbf{P}^{3}, \mathcal{I}_{Y}(r-1)\right)=0 .
$$

Proof. We start from the exact sequence

$$
0 \rightarrow \mathcal{I}_{Y \cup L} \rightarrow \mathcal{I}_{Y} \rightarrow \mathcal{I}_{Y / Y \cup L} \rightarrow 0
$$

where $\mathcal{I}_{Y / Y \cup L}=\mathcal{O}_{L}(-g)$. If we twist it with $\mathcal{O}_{\mathbf{P}^{3}}(r-1)$ and we pass to the long cohomology sequence, we get

$$
\begin{aligned}
0 & \rightarrow H^{0}\left(\mathbf{P}^{3}, \mathcal{I}_{Y \cup L}(r-1)\right) \rightarrow H^{0}\left(\mathbf{P}^{3}, \mathcal{I}_{Y}(r-1)\right) \rightarrow H^{0}\left(\mathbf{P}^{3}, \mathcal{O}_{L}(r-1-g)\right) \\
& \rightarrow H^{1}\left(\mathbf{P}^{3}, \mathcal{I}_{Y \cup L}(r-1)\right) \rightarrow H^{1}\left(\mathbf{P}^{3}, \mathcal{I}_{Y}(r-1)\right) \rightarrow H^{1}\left(\mathbf{P}^{3}, \mathcal{O}_{L}(r-1-g)\right) \\
& \rightarrow H^{2}\left(\mathbf{P}^{3}, \mathcal{I}_{Y \cup L}(r-1)\right) \rightarrow H^{2}\left(\mathbf{P}^{3}, \mathcal{I}_{Y}(r-1)\right) \rightarrow 0
\end{aligned}
$$

from which the result follows immediately.

Remark 4.2. This lemma means that we can remove from a stick-figure $\mathbf{Y}$ satisfying $H^{0}\left(\mathbf{P}^{3}, \mathcal{I}_{\mathbf{Y}}(r-1)\right)=H^{2}\left(\mathbf{P}^{3}, \mathcal{I}_{\mathbf{Y}}(r-1)\right)=0$ any $g$-secant, provided that $g \geq r$, conserving this property.

Our precise result is the following one:

Proposition 4.3. Fix an integer $m \geq 2$. For every integer $d, d_{m}<d \leq D_{m}$, there exists a smooth connected curve $X$, such that:

(1) $\operatorname{deg} X=d$,

(2) $H^{1}\left(X, \mathcal{N}_{X}(-1)\right)=0$,

(3) $H^{0}\left(\mathbf{P}^{3}, \mathcal{I}_{X}(m-1)\right)=H^{2}\left(\mathbf{P}^{3}, \mathcal{I}_{Y}(m-1)\right)=0$,

(4) $H^{1}\left(\mathbf{P}^{3}, \mathcal{I}_{X}(m-1)\right)=t$,

(5) $g(X)=G(d, m)-t$, where $t \in[0, t(d, m)]$ and $t(d, m)=p(2 r-1-p), p$ and $r$ defined as in $\S 3$.

Proof. (4) is a consequence of (3) and (5), by Riemann-Roch. As in Proposition 2.2 , we fix $r$ such that $d_{m(r-1)}<d \leq d_{m r}, 1 \leq r \leq \frac{m}{2}$, with the convention that $d_{m(m / 2)}=D_{m}$.

We consider again the stick-figure $\mathbf{X}_{m r}$ (defined in Remark 2.3). Let $f=d_{m r}-d$; note that if we write $d$ as $d=d_{m(r-1)}+p$ (as in $\S 3$ ), then $f=2 r-1-p$. We want to pick away $f$ lines from $\mathbf{X}_{m r}$. In order to have (3) it is enough to pick lines which are at least $m$-secants; in the diagram of $\mathbf{K}_{m}$, this means that the corresponding $\operatorname{dot}(i, j)$ satisfies $j \geq i+m-2 r+1$, i.e. it is above the dotted line having equation $y=x+m-2 r+1$. Moreover, if we remove an $(m+g)$-secant (instead of an $m$-secant as in $\S 2$ ), we remove $g$ more nodes. So, if we remove the $f$ disjoint lines

$$
L_{1 m-f+1}, L_{2 m-f+2}, \ldots, L_{f m}
$$


(which are $m+\left(2 r-1-f\right.$ )-secant lines to $\left.\mathbf{X}_{m r}\right)$, we get a stick figure $\mathbf{Y}$ with degree $d$ and arithmetic genus

$$
p_{a}=G(d, m)-f(2 r-1-f)=(2 r-1) p
$$

(note that this is exactly the number which bounds the $h^{1}\left(X, \mathcal{N}_{X}(-2)\right)$ of the curve with the same degree and maximal genus).

An easy check of Figure 7 shows that every genus between $G(d, m)-f(2 r-1-f)$ and $G(d, m)$ is actually possible. In fact, fix suitable injective functions $g: \underline{\mathbf{Z}}_{f} \rightarrow \underline{\mathbf{Z}}_{p}$ satisfying the condition $g(x) \geq x+m-2 r+1$ for every $x \in \underline{\mathbf{Z}}_{f}$, and then pick away the lines $L_{1 g(1)}, L_{2 g(2)}, \ldots, L_{f g(f)}$.

The vanishing $H^{1}\left(X, \mathcal{N}_{\mathbf{Y}}(-1)\right)=0$ is now a consequence of Lemma 2.1. Hence our stick-figure is smoothable, and by semicontinuity the smooth curve thus obtained also satisfies (1)-(5).

In a dual way the following proposition about the surjectivity of the map $\rho_{\mathbf{Y}}(m-1)$ can be proved:

Proposition 4.4. Fix an integer $m \geq 2$. For every integer $d, d_{m}<d \leq D_{m}$, there exists a smooth connected curve $X$ such that:

(1) $\operatorname{deg} X=d$,

(2) $H^{1}\left(X, \mathcal{N}_{X}(-1)\right)=0$,

(3) $H^{1}\left(\mathbf{P}^{3}, \mathcal{I}_{X}(m-1)\right)=H^{2}\left(\mathbf{P}^{3}, \mathcal{I}_{Y}(m-1)\right)=0$,

(4) $H^{0}\left(\mathbf{P}^{3}, \mathcal{I}_{X}(m-1)\right)=t$,

(5) $g(X)=G(d, m)+t$,

where $t \in\left[0, t^{\prime}(d, m)\right]$ and $t^{\prime}(d, m)=\left(\frac{m}{2}+1-r\right) f, f$ and $r$ as before.

\section{APPENDIX}

A. The construction of $[\mathrm{F}, \S 4.3]$ in terms of the diagrams. We describe via our diagrams a little variant of Fløystad's basic construction of [F, §4.3], showing, with the help of the diagrams, how it can be pushed a little further.

Fix $0 \leq t \leq \frac{m^{2}-p_{m}}{4}$; it is possible to pick away from $\mathbf{K}_{m} t$ lines, which at the step of their removal are $m$-subsecants, as explained in Proposition 2.2. Now, which lines are $(m-1)$-subsecants? We show in Figure 8 a strategy for picking away $s$ $(m-1)$-subsecants, which is successful whenever $s+t \leq \frac{m^{2}-p_{m}}{4}$, and even some more lines, as in the example below.

In the example below, $m=10, t=13, s=14$; hence $s+t>\frac{m^{2}}{4}$. The connectedness and the smoothability can be checked directly in Figure 8.

B. About a claim of Halphen. A careful study of the construction of Fløystad also gives the proof of an ancient claim of Halphen: namely, there exist curves of degree $d$ and genus $g$ contained in surfaces of minimal degree $m-1$, for every $g$, $G(d, m)<g \leq G(d, m-1)$. 


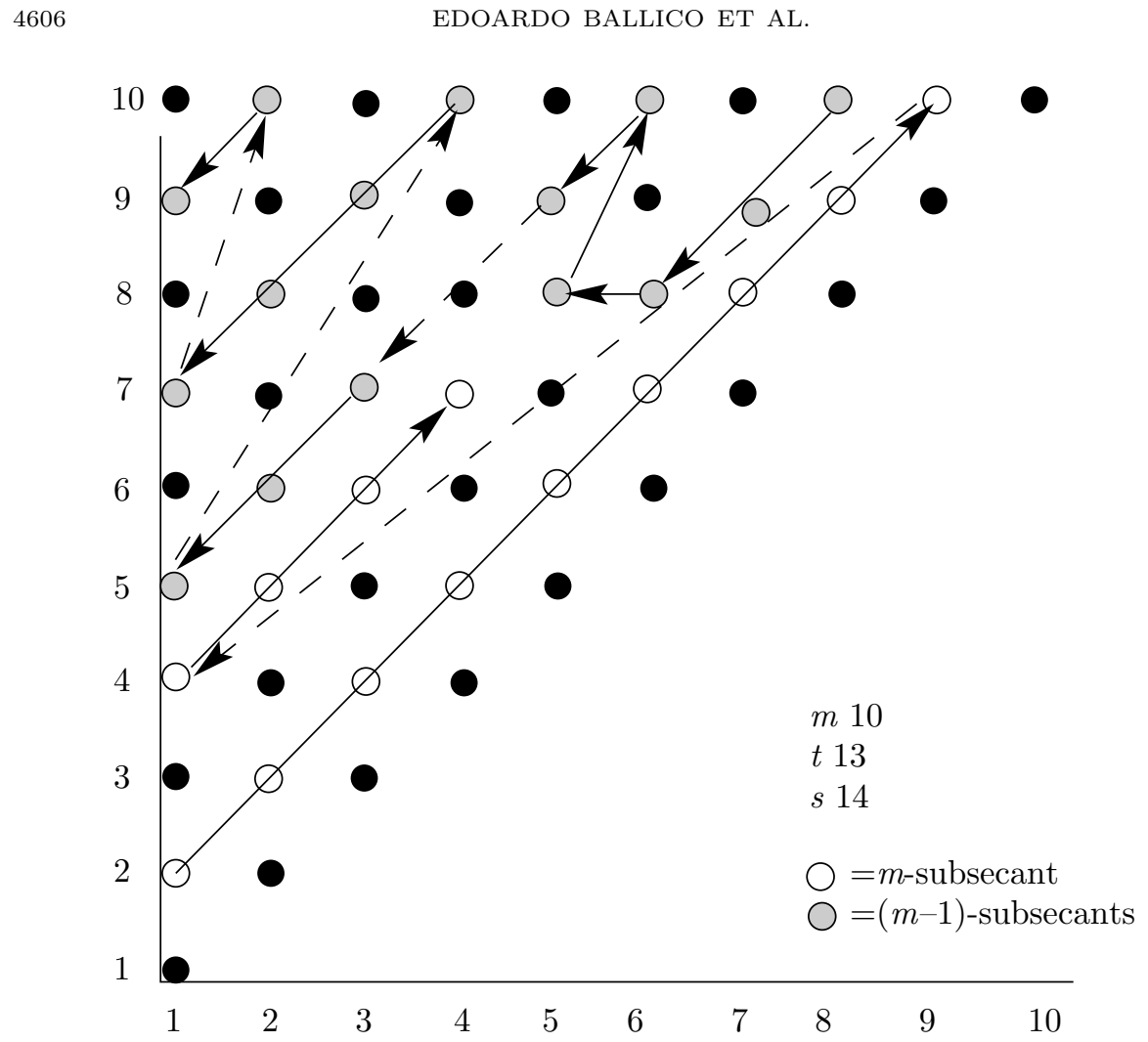

Figure 8

This can be seen easily as follows. Consider the $(d, g)$ plane; let us denote as before

$$
\begin{aligned}
D_{m}=\operatorname{deg} \mathbf{K}_{m}=\frac{m^{2}+m}{2} \quad\left(\text { in Fløystad's notation, } d_{m}\right), \\
g_{m}=p_{a}\left(\mathbf{K}_{m}\right)=\frac{2 m^{3}-3 m^{2}-5 m+6}{6} ; \quad P_{m}=\left(D_{m}, g_{m}\right), \\
d_{m}=\frac{(m+1)^{2}-o_{m}}{4}=\text { degree of the curve obtained from } \mathbf{K}_{m} \text { by picking } \\
\text { away } \left.\frac{m^{2}}{4}-o_{m} \text { lines (in Fløystad's notation, } d_{m}^{\prime}\right),
\end{aligned}
$$

$g_{m}^{1}=$ degree of the curve obtained from $\mathbf{K}_{m}$ by picking away $\frac{m^{2}}{4}-o_{m}$ $(m-1)$ subsecants,

$g_{m}^{2}=$ degree of the curve obtained from $\mathbf{K}_{m}$ by picking away $\frac{m^{2}}{4}-o_{m}$ $(m)$ subsecants,

$P_{m}^{1}=\left(d_{m}, g_{m}^{1}\right), \quad P_{m}^{2}=\left(d_{m}, g_{m}^{2}\right)$. 
Fløystad's construction gives stick-figures of degree $(d, g)$ obtained from $\mathbf{K}_{m}$ for any $(d, g)$ in the triangle $\left\langle P_{m}, P_{m}^{1}, P_{m}^{2}\right\rangle$. Those stick-figures corresponding to the $P_{m} P_{m}^{2}$ side are exactly the curves of Proposition 2.2 (obtained by picking away $m$ subsecants); hence the segment $P_{m} P_{m}^{2}$ lies on the line of equation $g=G(d, m)$, as already noted; more generally, $P_{m-1}^{2}, P_{m}^{1}, P_{m-1}$ and $P_{m}$ lie on the line of equation $g=G(d, m-1)$ (this is a direct and easy check).

A stick-figure $X$ corresponding to a point of the triangle $\left\langle P_{m}, P_{m}^{1}, P_{m}^{2}\right\rangle$ not on the side $P_{m} P_{m}^{2}$ has

$$
H^{1}\left(\mathbf{P}^{3}, \mathcal{I}_{X}(m-1)\right)=H^{2}\left(\mathbf{P}^{3}, \mathcal{I}_{Y}(m-1)\right)=0,
$$

and

$$
H^{0}\left(\mathbf{P}^{3}, \mathcal{I}_{X}(m-1)\right) \neq 0
$$

since it is obtained by picking away at least one $(m-1)$-subsecant (apply Lemma $1.5)$.

So, as a corollary to Fløystad's construction and to the discussion above, we have that for every $d, d_{m} \leq d \leq D_{m}$ and for every $g, G(d, m)<g \leq G(d, m-1)$ there exists a stick-figure of degree $d$ and genus $g$ contained in surfaces of degree $m-1$, such that

$$
\begin{aligned}
H^{1}\left(\mathbf{P}^{3}, \mathcal{I}_{X}(m-1)\right) & =H^{2}\left(\mathbf{P}^{3} \mathcal{I}_{Y}(m-1)\right)=0 \\
& =H^{1}\left(X, \mathcal{N}_{X}(-1)\right) .
\end{aligned}
$$

By semicontinuity, it specializes to a smooth curve with the same properties.

\section{REFERENCES}

[BE] E. Ballico and Ph. Ellia, A program for space curves, Conf. Algebraic Varieties of Small Dimension, Rend. Sem. Mat. Univ. Politec. Torino, special issue (1986), 25-42. MR 89e:14031

[E] Ph. Ellia, Sur le genre maximal des courbes gauches de degré d non sur une surface de degré s - 1, J. Reine Angew. Math. 413 (1991), 78-87. MR 92a:14029

[ES] Ph. Ellia and R. Strano, Sections planes et majoration du genre des courbes gauches, Complex Projective Geometry, London Math. Soc. Lecture Note Ser., vol. 179, Cambridge Univ. Press, Cambridge and New York, 1992, pp. 157-174. MR 94b:14028

[F] G. Fløystad, Construction of space curves with good properties, Math. Ann. 289 (1991), 33-54. MR 92h:14021

[GP1] L. Gruson and Chr. Peskine, Genre des courbes de l'espace projectif, Algebraic Geometry (Troms $\varnothing, 1977$ ), Lecture Notes in Math., vol. 687, Springer-Verlag, New York and Berlin, 1978, pp. 31-59. MR 81e:14019

[GP2] _ Postulation des courbes gauches, Algebraic Geometry (Ravello, 1982), Lecture Notes in Math., vol. 997, Springer-Verlag, New York and Berlin, 1983, pp. 218-227. MR 85f: 14029

[H] G. H. Halphen, Mémoire sur la classification des courbes gauches algébriques, Ouvres Completes, t. III, pp. 261-455.

[Ha1] R. Hartshorne, On the classification of algebraic spaces curves II, Proc. Sympos. Pure Math., vol. 46, Amer. Math. Soc., Providence, RI, 1987, pp. 145-164. MR 89g:14025

[Ha2] Stable reflexive sheaves III, Math. Ann. 279 (1988), 517-534. MR 89h:14011

[HH1] R. Hartshorne and A. Hirschowitz, Smoothing algebraic space curves, Algebraic Geometry (Sitges, 1983), Lecture Notes in Math., vol. 1124, Springer-Verlag, New York and Berlin, 1985, pp. 98-131. MR 87h:14023

[HH2] _ Nouvelles courbes de bon genre dans l'espace projectif, Math. Ann. 280 (1988), 353-367. MR 89d:14043 
[S1] R. Strano, Plane sections of curves of $\mathbf{P}^{3}$ and a conjecture of Hartshorne and Hirschowitz, Rend. Sem. Mat. Univ. Politec. Torino 48 (1990), 511-527. MR 94e:14041

[S2] - On the genus of a maximal rank curve in $\mathbf{P}^{3}$, J. Algebraic Geom. 3 (1994), 435447. MR 95c: 14031

Dipartimento di Matematica, Università di Trento, I-38050 Povo (Trento), Italy

E-mail address: ballico@science.unitn.it

Istituto di Matematica e Fisica, Università di Sassari, Via Vienna 2, I-07100 Sassari, ITALY

E-mail address: bolondi@science.unitn.it

Dipartimento di Matematica, Università di Ferrara, Via Machiavelli 35, I-44100 FerRARA, ITALY

E-mail address: phe@dns.unife.it

Departimento Algebra y Geometrìa, Facultad de Matemàticas, Universidad de BarCELONA, 08007 BARCElona, Spain

E-mail address: miro@cerber.ub.es 